\title{
Motoneuron firing and isomyosin type of muscle fibres in prior polio
}

\author{
KRISTIAN BORG, JÖRGEN BORG,* GURTEJ K DHOOT, $\dagger$ LARS EDSTRÖM, \\ LENNART GRIMBY, LARS-ERIC THORNELL \\ From the Departments of Neurology, Karolinska Sjukhuset and Södersjukhuset, ${ }^{*}$ Stockholm, Sweden, \\ Department of Immunology, $\dagger$ University of Birmingham, United Kingdom, and Department of Anatomy, $\ddagger$ Umea \\ University, Umeå, Sweden
}

SUMMARY In patients with prior polio there was an excessive use of remaining motor units and an absence of type II muscle fibres in the tibialis anterior (TA). In the present study, eight subjects with prior polio with more than $90 \%$ type I fibres in the TA were examined. The aim was to elucidate whether the lack of type II muscle fibres was due to a selective loss of motoneurons with high threshold and high axonal conduction velocity or due to a muscle fibre transition from type II to type I. There was no decrease of the proportion of motoneurons with high threshold and high axonal conduction velocity. Monoclonal antibodies against fast and slow myosin heavy chains (MHC) were used as histochemical markers and many muscle fibres of type I according to ATPase stainability showed a binding of both anti-fast and anti-slow MHC. It is suggested that the type I muscle fibre dominance in prior polio subjects with excessive use of TA during walking is due to a muscle fibre transition from type II to type I and not to a loss of one class of motor units.

Normally about $75 \%$ of the fibres in the human musculus tibialis anterior (TA) are type I and $25 \%$ type II. TA is used mainly during locomotion; type I fibres are used several times in each step cycle; type II fibres are used only during rapid locomotion or rapid corrective movements and then usually only a few times. ${ }^{1}$ In patients with long-lasting moderately severe lower motoneuron disorders all remaining TA units were found to be used tonically in each step cycle to compensate for the loss of power and there were none or hardly any type II TA fibres, as revealed by enzyme histochemistry. ${ }^{2}$ In patients with such severe disorders that the remaining TA units were useless for locomotion, however, the differentiation into type I and II muscle fibres remained.

In normal animals motoneurons innervating fasttwitch muscle fibres differ from those innervating slow-twitch fibres by a higher density of large inhibiting C-boutons at the axonal hillock, a larger dendritic tree, a lower input resistance, a shorter afterhyperpolarisation, a higher threshold for activation, a

Correspondence to: Kristian Borg, Department of Neurology, Karolinska Sjukhuset, S-104 01 Stockholm, Sweden.

Received 23 December 1988.

Accepted 22 March 1989 higher firing rate and a higher axonal conduction velocity. The last three parameters have been studied in human experiments and correlations to muscle fibre type have been demonstrated also in humans (for references see review by Edström and Grimby $1986^{3}$ ).

In animal experiments overuse evoked by long-term electrical tetanisation caused a transition of type II to type I muscle fibres. ${ }^{4}$ The myosin ATPase content and the properties of myosin light and heavy chains of fasttwitch muscle fibres changed to the pattern typical of slow-twitch fibres. ${ }^{45}$ During the muscle fibre transition both fast and slow myosin occurred simultaneously. ${ }^{6}$ In our cases with type I fibre dominance, the enzyme histochemistry did not offer any obvious signs of heterogeneity within the type I fibre group. ${ }^{2}$ It is, however, not self-evident that the type I fibres in the excessive overusers are homogenous with reference to other myosin properties than ATPase stainability, and thus a further analysis of the type I fibres with immunohistochemical markers for fast and slow myosin isoforms is relevant for the question of fibre transformation.

In the present study the properties of remaining TA motoneurons and muscle fibres in patients almost lacking type II TA fibres were compared with those of normal subjects. The aim was to elucidate whether the absence of type II muscle fibres was due to a loss of 
motoneurons with high threshold and high axonal conduction velocity or to a transition of muscle fibres innervated by such motoneurons.

\section{Subjects and methods}

Eight subjects (five women, three men) with TA paralysis due to prior poliomyelitis, with at least $90 \%$ (mean $96.7 \%$, range $90 \cdot 7 \%-100 \%$ ) type I muscle fibres in TA as determined by ATPase stainability, were examined. Their mean age at the examination was 58 years (range 37-71) and the mean age at the acute onset of polio was 21 years (range 5-48). The mean time between the acute infection and the examination was 37 years (range 21-51).

\section{Electromyographic recordings}

In subjects with prior polio, with a decreased number of motor units and increased muscle fibre density within remaining units, single motor units were easily obtained by ordinary EMG needle electrodes. Also in normal subjects there were occasional clusters of muscle fibres innervated by one and the same motoneuron, possibly as a result of the normal denervation-reinnervation processes. When a highly selective needle or wire electrode was inserted into such a cluster, recordings in which only one motor unit had a high amplitude potential during maximal voluntary tension could be obtained. The techniques are described in detail elsewhere. $^{78}$ The risk of confusing potentials from different motor units was small because of the characteristic shape of reinnervated motor units. Single motor unit recordings could be verified by observing that the voluntary test unit potential was identical to that evoked by supramaximal electrical stimulation of the nerve to the muscle. The surface EMG was recorded simultaneously. The signals were rectified and integrated in a linear way over periods of $80-320 \mathrm{~ms}$ and displayed during the next period (compare fig 1,2 ).

The velocity of the most rapidly conducting axons was calculated by means of measuring the F-wave latency. ${ }^{9}$ The deep peroneal nerve was supramaximally stimulated at the knee and the M-response and F-wave were recorded with a $\vec{\circ}$ Medelec surface electrode (no. C 162 Medelec Ltd, Woking,

Fig 1 Polio subject, upper trace shows simultaneous recordings of three TA motor units during slowly increasing voluntary tension. Time bar I s. For further description see Results.

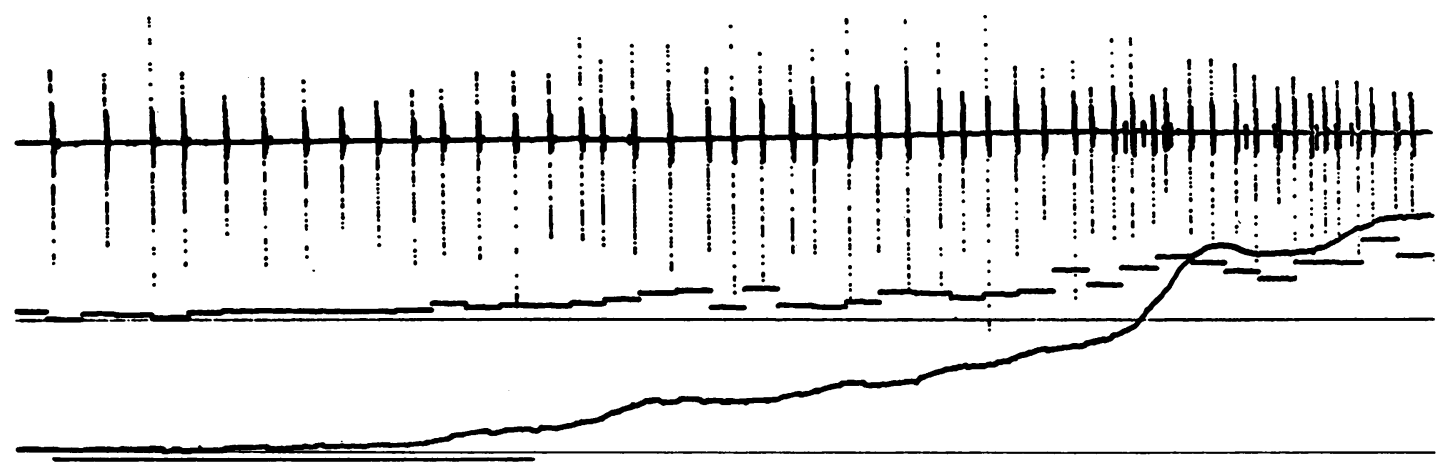

Fig 2 Polio subject, simultaneous recordings of a low threshold TA motor unit (high amplitude potential), and one high threshold unit recruited at about $20 \mathrm{~Hz}$ (low amplitude potential). Lower traces show surface TA EMG integrated over periods of $80 \mathrm{~ms}$ and displayed during the following period and the voluntary tension of foot dorsiflexors. Time bar $1 \mathrm{~s}$. For further description see Results. 
Surrey, UK). The shortest F-wave latency (F latency-M latency) that could be reproduced 10 times was used for calculation. The distance between the lower border of the Th 12 spinous process and the stimulation point was measured and the motor nerve conduction velocity calculated. Data were compared with data from 10 healthy volunteers (mean age 50 years, range $30-64$ ).

The axonal conduction velocity of a single motor unit, calculated from the latency difference of its potential after proximal and distal electrical stimulation of the peroneal nerve, ${ }^{10}$ could not be studied in a conclusive way in the TA since the distance between suitable stimulation points was too short. The axonal conduction velocity and its relation to the voluntary firing pattern could, however, easily be studied in the extensor digitorum brevis (EDB). Ten prior polio subjects with only a few remaining EDB units were studied. Stimuli were delivered through $6 \mathrm{~mm}$ diameter surface electrodes. The cathode was placed over the nerve and the position was adjusted so that minimum stimulus strength was required for muscle response. The anode was placed $2-3 \mathrm{~cm}$ medial or lateral to the cathode. Rectangular pulse waves of $0.2 \mathrm{~ms}$ duration were used. Stimulus strength could be continuously varied between 0 and $100 \mathrm{~mA}$. The skin temperature was kept constant at $32^{\circ} \mathrm{C}$ by means of a Disaheater (DISA Electronic, Skovlunde, Denmark).

\section{Muscle biopsy procedure and tissue processing}

TA muscle biopsies were performed by the percutaneous conchotome method originally described by Radner ${ }^{11}$ and slightly modified. ${ }^{12}$ The percutaneous muscle biopsy technique was used for practical and ethical reasons. The biopsy site was carefully selected and such areas where some contraction could be felt through the skin were chosen for biopsy trials.

The material aimed for light microscopical morphology and histochemistry was quickly frozen in Freon 13 kept at its melting point $\left(-190^{\circ} \mathrm{C}\right)$ by liquid nitrogen and kept in a refrigerator at $-75^{\circ} \mathrm{C}$ until further processed. Sections of $6-15 \mu \mathrm{m}$ were cut in a cryostat operating at $-25^{\circ} \mathrm{C}$.

\section{Enzyme histochemical methods and fibre type nomenclature} The muscle fibre nomenclature was based on the stainability for adenosine triphosphatase (ATPase). The ATPase staining was performed according to the original method of Padykula and Herman ${ }^{13}$ with the modification by Brooke and Kaiser. ${ }^{14}$ Thus, fibres with high content of acid-stable ATPase and low content of alkali-stable ATPase were termed "type I" while fibres with the reversed pattern of ATPase stainability were termed "type II". Fibres with an intermediate stainability for ATPase that is, with varying greyness after alkaline and acid preincubation were termed "intermediate (IM)", (compare Schantz and Dhoot). ${ }^{15}$ All fibres on a muscle biopsy cross-section were classified and the total number of fibres of each type estimated.

\section{Immunohistochemical methods}

Monoclonal antibodies to slow and fast skeletal myosin heavy chains (MHC), respectively, stained and tested as previously described, ${ }^{11} 16$ were used. The antibodies against slow MHC have been shown to bind only to type I fibres in adult human triceps muscle, whereas the antibodies against fast MHC only bind to type II fibres. ${ }^{1516}$ Fibres which contained both slow and fast MHC were called "promiscuous (P)". The antigens in the cryostat serial sections were located by the immunoperoxidase sandwich labelling PAPtechnique. ${ }^{17}$ Sections were examined in an Olympus Vanox or a Leitz Orthoplan light microscope.

\section{Results}

\section{Motoneuron properties}

When normal subjects slowly increased voluntary tension the TA units with the lowest thresholds were recruited at zero tension and attained tonic firing at regular intervals before $10 \mathrm{~Hz}$ (SD of 10 consecutive intervals was less than $10 \mathrm{~ms}$ ). The units with the highest thresholds, on the other hand, were not recruited until $80-90 \%$ of maximal tension and did not attain regular firing before $20 \mathrm{~Hz}{ }^{7}$ Most units had, however, intermediate properties. A motor unit with a higher threshold fired at lower rates than a unit with a lower threshold until it attained regular firing intervals. After this critical level of contraction both type of units fired at similar rates except for maximal effort, when high threshold units tended to fire at higher rates than low threshold units (for further normal data see references ${ }^{101819}$ ).

To get a hint of the relative number of low and high threshold TA units, the firing rate of one low threshold unit was compared with the surface EMG during isometric voluntary tension slowly increasing from zero to maximum. Recruitment of new motor units then appeared as a more rapid increase of the surface EMG than of the firing rate of the test unit. In normal subjects TA units fulfilling the test unit criteria were abundant. The global EMG increased two to three times when the firing rate of the test unit increased from 10 to $15 \mathrm{~Hz}$, two to three times from 15 to $20 \mathrm{~Hz}$, and about two times from 20 to $35 \mathrm{~Hz}$. These findings indicate a recruitment of new motor units during most of the firing range of the test unit with a peak about $15 \mathrm{~Hz}$.

In each one of the polio subjects there was also a continuum between low threshold units firing at regular intervals at $10 \mathrm{~Hz}$ and high threshold units not firing regularly before $20 \mathrm{~Hz}$. Figure 1 shows three single TA units during slowly increasing isometric voluntary contraction. The unit with the lowest threshold (low amplitude potential) attained 15-20 Hz before the other two units were recruited. The unit with moderately high threshold (intermediate amplitude potential) fired doublets at recruitment and attained regular firing intervals at about $20 \mathrm{~Hz}$.

TA units fulfilling the test unit criteria were scarce. Consequently the global EMG and tension was often not measurable at $10 \mathrm{~Hz}$. The global EMG increased rapidly at about $15 \mathrm{~Hz}$ and was at $20 \mathrm{~Hz}$ two to five times that recorded at $15 \mathrm{~Hz}$. Consequently, tension increased rapidly after $15 \mathrm{~Hz}$. Figure 2 illustrates the 
findings in one polio patient lacking type II fibres. When the firing rate of the low threshold test unit (high amplitude potential upper trace) was lower than $15 \mathrm{~Hz}$ the surface EMG was less than $10 \%$ of maximum and tension very low. Between 15 and $20 \mathrm{~Hz}$ there was a more rapid increase of the surface EMG than the firing rate. The findings indicate that a large proportion of the TA units were not recruited before $15 \mathrm{~Hz}$.

A selective loss of TA units with high axonal conduction velocity should result in a slowing of the maximal conduction velocity of the nerve to the muscle. The shortest latency in a series of F-wave responses has been used to study the TA conduction velocity' since each motor unit had its particular Fwave latency supposed to be due largely to its conduction velocity. In polio subjects $\mathrm{F}$-waves were easily obtained. The shortest mean F-wave latency was the same, $\mathbf{3 4} \mathrm{ms}$, in the polio subjects and in the controls and the motor nerve conduction velocity in the peroneal nerve was calculated to $45 \mathrm{~m} / \mathrm{s}$ in the polio subjects and $44 \mathrm{~m} / \mathrm{s}$ in the normal controls.

As mentioned above, the axonal conduction velocity of single motor units could be studied systematically only in the EDB muscle. In this muscle, as well as in TA, most motor units were lost. However, a continuum remained between motor units firing at regular intervals below $10 \mathrm{~Hz}$ and motor units firing regularly only above $20 \mathrm{~Hz}$. Figure $3 \mathrm{~A}$ shows that the

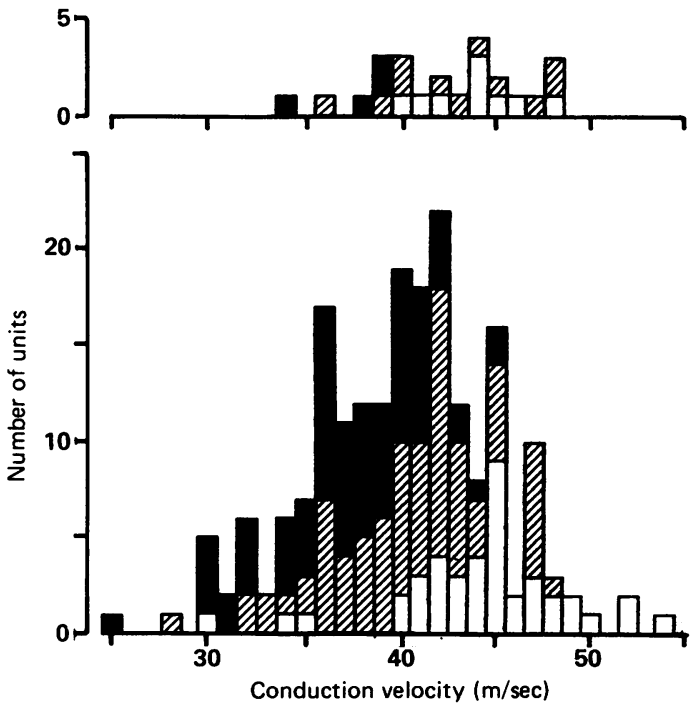

Fig 3 Axonal conduction velocities and voluntary discharge patterns in 24 motor units of the extensor digitorum brevis $(E D B)$ muscle in prior polio $(A)$ and in $200 E D B$ units from normal controls $(B)$. Solid bars are motor units with low threshold, open bars high threshold and hatched bars are motor units with intermediate threshold. axonal conduction velocities of $24 \mathrm{EDB}$ units from the 10 polio subjects ranged between 34 and $48 \mathrm{~m} / \mathrm{s}$. Low threshold units with minimal firing rates of less than $10 \mathrm{~Hz}$ had conduction velocities between 34 and $39 \mathrm{~m} / \mathrm{s}$ while high threshold units with minimal rates of more than $20 \mathrm{~Hz}$ had velocities between 40 and $48 \mathrm{~m} / \mathrm{s}$. Figure 3B shows that the axonal conduction velocities of $200 \mathrm{EDB}$ units from 20 normal subjects ranged between 30 and $55 \mathrm{~m} / \mathrm{s}$ and that low threshold units had axonal conduction velocities between 30 and $45 \mathrm{~m} / \mathrm{s}$, and high threshold units between 40 and $55 \mathrm{~m} / \mathrm{s}$.

\section{Muscle biopsy findings}

A number of pathological features were observed in the muscle biopsy specimens including diversity in fibre sizes: atrophy and hypertrophy, fibre splitting, internal nuclei, rimmed vacuoles, etc. (For details see reference ${ }^{2}$ ). A vast majority of the fibres showed low ATPase activity at pH $\mathbf{9 . 4}$ and high activity after preincubation at pH 4.3 (type I fibres), (table, figs 4A, B and 5A, B). The few type II fibres (with high ATPase activity at $\mathrm{pH} 9.4$ and low after preincubation at $\mathrm{pH}$ 4.3) were single or formed small groups. Rare fibres showed intermediate ATPase activity (IM fibres, figo 4A and 5A). The mean percentage of type I, II and IMP fibres is presented in the table. The number of type $B$ fibres was significantly higher than in normal $T \overrightarrow{\mathbb{C}}$ muscles (references ${ }^{12}$ ). A majority of type I fibres were large in comparison with normal and considered as hypertrophic but a small number of moderatel 8 atrophic fibres were found. Groups of highly atrophie fibres containing both fibre types were found as show in fig 6.

Table Percentage of fibres stained for myofibrillar ATPase after acid and alkaline incubation and fibres labelled with antibodies against slow and fast myosin heavy chains (MHC) in biopsy samples of overusers

\begin{tabular}{|c|c|c|c|c|c|c|}
\hline \multirow[b]{2}{*}{ Case } & \multicolumn{3}{|c|}{ Fibre types } & \multicolumn{3}{|c|}{$\begin{array}{l}\text { Fibres positively stained } \\
\text { with myosin antibodies }\end{array}$} \\
\hline & $I$ & $I M$ & $I I$ & $M H C(s)$ & $M H C(f)$ & $P$ \\
\hline $\begin{array}{l}1 \\
2 \\
3 \\
4 \\
5 \\
6 \\
7 \\
8 \\
\text { Mean } \\
\text { SD }\end{array}$ & $\begin{array}{c}94 \\
95.8 \\
90.7 \\
97.9 \\
99 \\
100 \\
99.3 \\
96.6 \\
96.7 \\
3.1\end{array}$ & $\begin{array}{l}3 \cdot 0 \\
0.3 \\
2 \cdot 2 \\
0.3 \\
0.6 \\
0 \\
0 \\
0.4 \\
0.9 \\
1 \cdot 1\end{array}$ & $\begin{array}{l}3.0 \\
3.9 \\
7.1 \\
1.9 \\
0.4 \\
0 \\
0.7 \\
2.9 \\
2.5 \\
2.3\end{array}$ & $\begin{array}{l}84.6 \\
93.7 \\
76.3 \\
92.2 \\
96.7 \\
98.8 \\
91.6 \\
90 \\
90.6 \\
7.3\end{array}$ & $\begin{array}{l}2.6 \\
3.5 \\
3.7 \\
0.3 \\
0.3 \\
0 \\
0 \\
0 \\
1.3 \\
1.7\end{array}$ & $\begin{array}{r}12 \cdot 8 \\
2 \cdot 8 \\
20.1 \\
7 \cdot 2 \\
3.0 \\
0.2 \\
8.4 \\
10 \\
8.1 \\
6.4\end{array}$ \\
\hline
\end{tabular}

I, fibres with low ATPase activity at $\mathrm{pH} 9.4$ and high after preincubation at $\mathrm{pH} \mathrm{4 \cdot 3}$. II, fibres with reversed pattern. IM, fibres with intermediate characteristics. MHC(s), fibres labelled against slow MHC. MHC(f), fibres labelled against fast MHC. P, promiscuous fibres labelled with both antibodies against slow and fast MHC. 

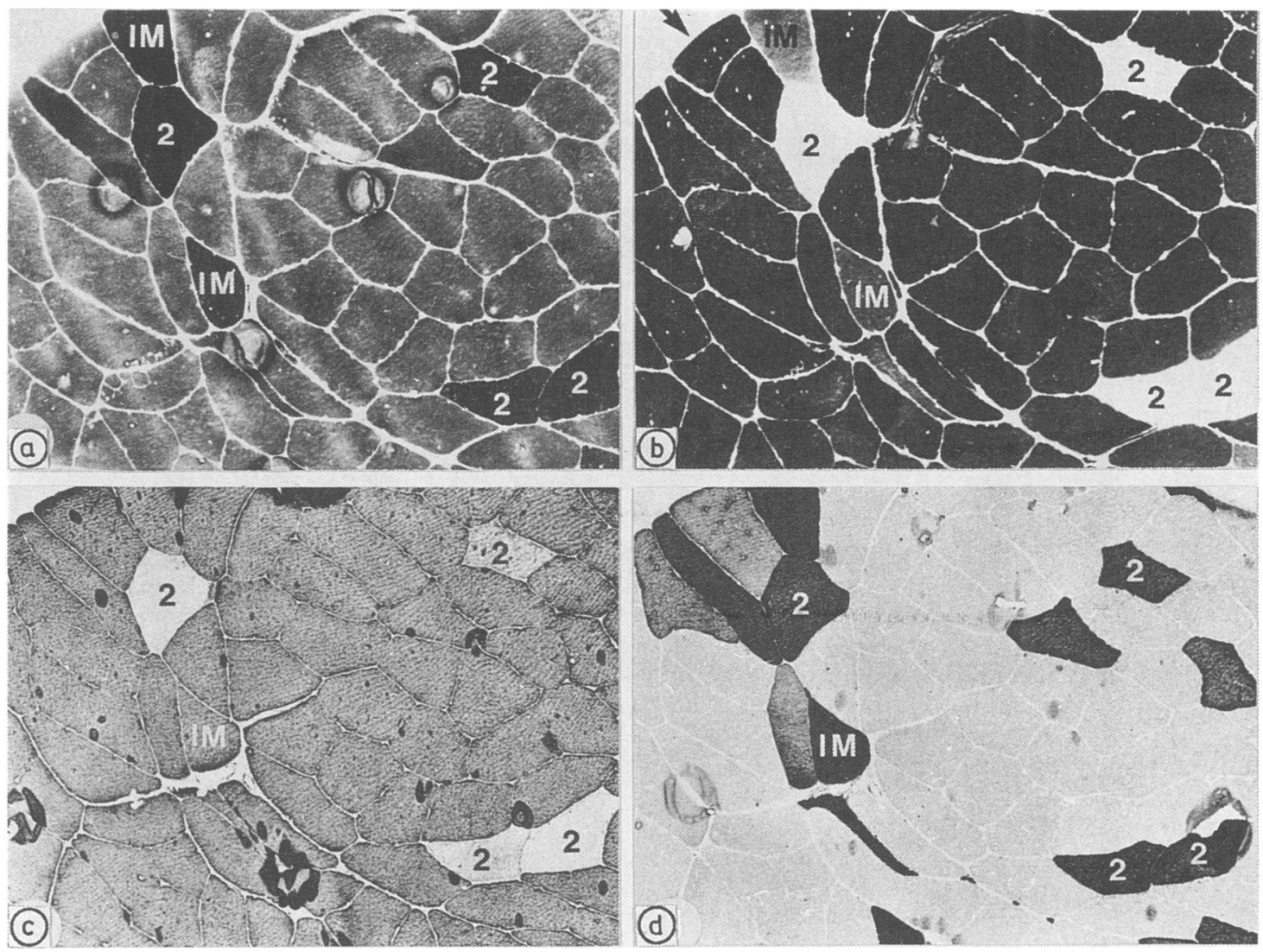

Fig $4(\times 80)$ Serial cross-sections of a biopsy specimen from TA muscle. A. Myofibrillar ATPase at pH 9.4. B. Myofibrillar ATPase after preincubation at pH 4.3. C. Anti-slow MHC. D. Anti-fast MHC. A and B. The majority of the fibres show type I fibre characteristics (low ATPase activity at pH 9.4 and high after acid preincubation). Four fibres (2) show' type II characteristics (high activity at $\mathrm{pH} 9.4$ and low after acid preincubation) and some fibres show intermediate ATPase activity at pH 9.4 and various degrees of staining after preincubation at pH 4.3 (IM). Several fibres contain internal nuclei. One split fibre is seen (arrow in B, compare with $A, C$ and D). C. All fibres except the four type II fibres show strong staining. Note however that two of the type II fibres are not completely unstained. D. Several fibres are stained. Note various degrees of staining in fibres of type II, IM and type I.

In the sections stained with anti-slow MHC, nearly all fibres showed a staining reaction and only few were unreactive (fig 4C, 5C). In one case, groups of unreactive fibres were observed. In sections labelled with anti-fast MHC a minority of the fibres showed a staining. The number of reactive fibres were, however, in all biopsy specimens higher than that of the fibres unreactive for anti-slow MHC (table). These were either of normal size, large or small. Furthermore, they showed various shades of reactivity (figs 4D, 5D).

By comparison of serial sections it was clear that several fibres of normal or large size were reactive for both anti-slow MHC and anti-fast MHC, so called promiscuous fibres $(\mathrm{P}$, table). In sections stained for myofibrillar ATPase these promiscuous fibres were typical type I, II or IM fibres (figs 4, 5).

\section{Discussion}

In normal human subjects the type of motoneuron can be identified by its minimal firing rate during voluntary contraction and its axonal conduction velocity. In prior polio subjects, the proportion of motor units with high minimal firing rate and high axonal conduction velocities, was not decreased. ${ }^{20}$ This finding suggests that the absence of type II muscle fibres was not due to a selective loss of large motoneurons. However, both parameters used for motoneuron 

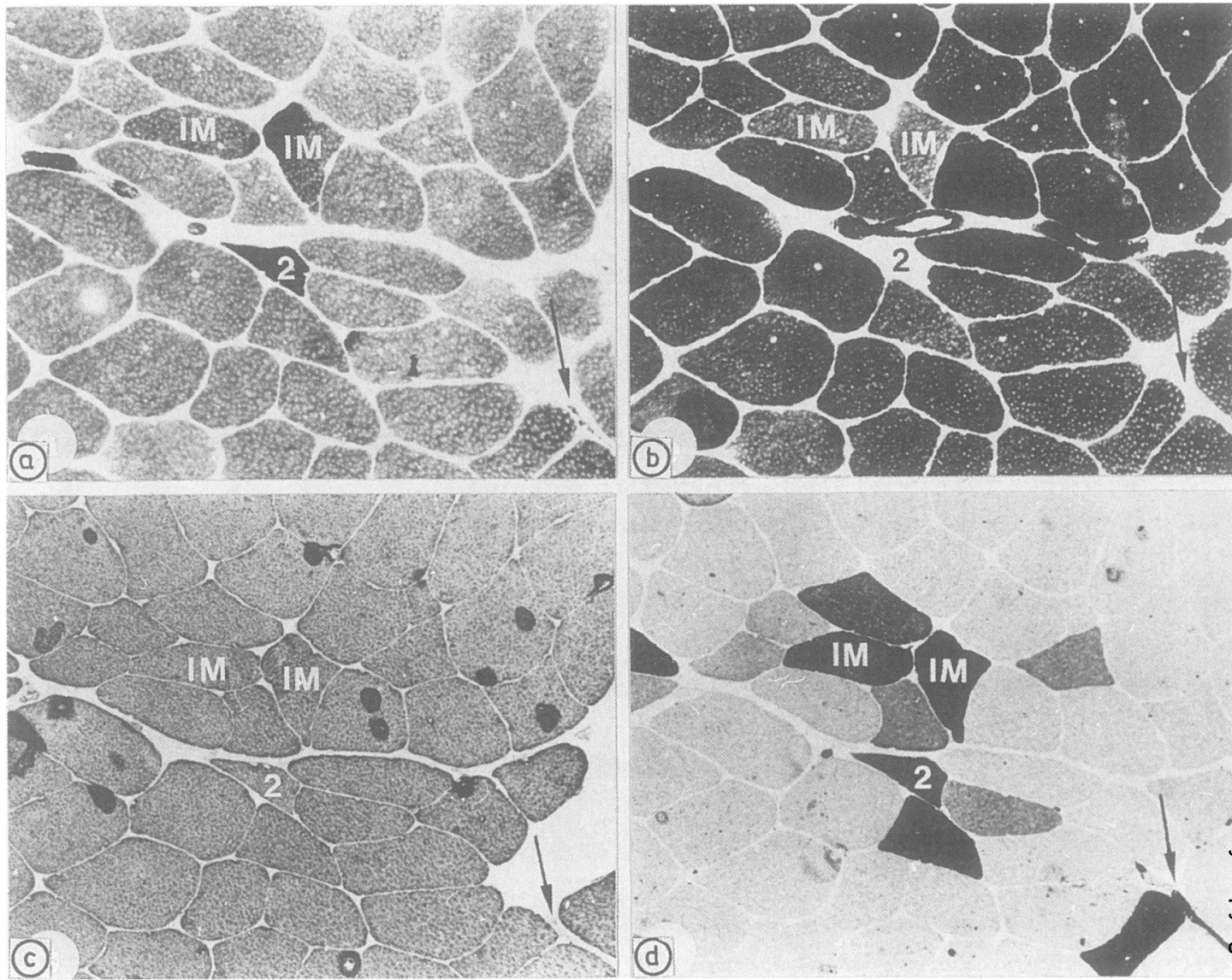

Fig $5(\times 80)$ Serial cross-sections of a biopsy specimen from TA muscle. A. Myofibrillar ATPase 9.4. B. Myofibrillar ATPase after incubation at $\mathrm{pH} 4 \cdot 3$. C. Anti-slow MHC. D. Anti-fast MHC. A and B. Only one fibre shows high ATPase activity at pH 9.4 (2). That fibre and a small slender fibre (arrow) show no ATPase activity, after preincubation at pH $4 \cdot 3$. Some fibres show an intermediate ATPase activity at $\mathrm{pH} 9.4$ and a somewhat inhibited ATPase activity after preincubation at pH 4.3 (IM). C. All fibres except for the small slender fibre (arrow) are stained for slow MHC. D. Several fibres show staining for fast MHC, including the type II fibre (2), the small slender fibre (arrow) and the IM fibres (IM).

identification might be disturbed by pathological processes of the motoneuron pool; the after-hyperpolarisation, determining the minimal firing rate, decreased in intact soleus motoneurons after partial denervation of the muscle $;^{21}$ the synaptic properties changed on axotomy;22 the dendritic properties changed on chronic stimulation, ${ }^{23}$ the voluntary motoneuron firing changed with the contractile properties of the muscle $;^{24}$ the axonal conduction might increase with increasing number of innervated muscle fibres. ${ }^{25-27}$

Experimental data imply that the amount of impulses govern the type characteristics-that is, the phenotypic expression of the muscle fibres. ${ }^{28} 29$ With respect to the histochemical staining for myofibrillar ATPase this is directly related to the expression of slow and fast myosin heavy chains, respectively (references ${ }_{530}$ ). That is, for induction of slow myosin heavy chains frequent or continuous stimulation of muscle fibres are needed ${ }^{5}$ which corresponds to the activity pattern of small slow-twitch, low threshold motoneurons.

All available data suggest that during everyday life in normal subjects, the motoneurons with the lowest thresholds fire at low rates for long periods of time and innervate slow-twitch-type I muscle fibres. Neurons with high thresholds fire in scarce and short high frequency bursts and innervate fast-twitch, fast fatiguing muscle fibres-type IIB. Neurons with intermediate firing and threshold properties innervate fast-twitch, fatigue resistant fibres-type IIA (for review see Edström and Grimby $1986^{3}$ ).

It has been difficult to reproduce muscle fibre 


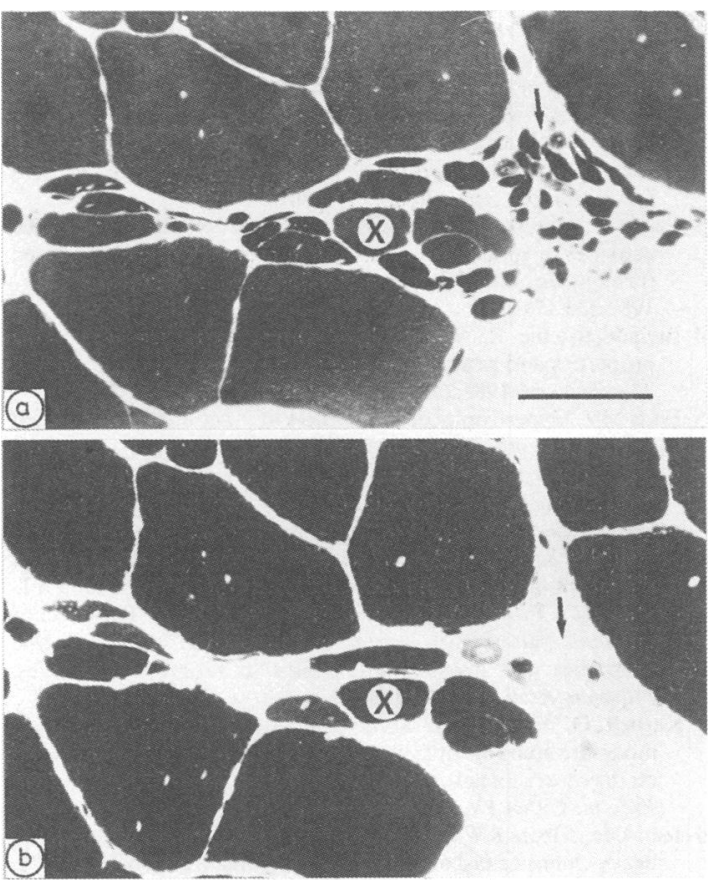

Fig 6 Serial cross-sections of a biopsy specimen from $T A$ muscle stained for myosin ATPase at pH 9.4 (a) and pH 4.3 (b). Note that all large sized fibres are of type I (darkly stained in b). A cross indicates an atrophic fibre of type I and an arrow indicates a group of highly atrophic fibres containing fibres of type I as well as of type II (unstained in b). Bar in a indicates $50 \mu \mathrm{m}$.

transformation in voluntary exercise in normal humans. Larsson and Ansved ${ }^{31}$ found that in elite rowers, the number of fast-twitch (type II) fibres increased and slow-twitch (type I) fibres decreased during a detraining period. Schantz et al, ${ }^{32}$ Schantz and Henriksson, ${ }^{33}$ and Schantz and Dhoot ${ }^{15}$ demonstrated that after extreme cross-country ski training there was an increase of ATPase intermediate fibres in triceps muscle which contained variable amounts of fast and slow isoforms of myofibrillar proteins in the same fibres. They presumed that these fibres represented transitional forms indicating fibre type transformation.

In patients with lower motoneuron disorders causing moderately severe paralysis, all remaining motor units fired at about $40 \mathrm{~Hz}$ for half of the step cycle during ordinary walking, resulting in full fused contraction for considerable parts of the day and for a very long period of life. Thus, in these patients the pattern of transneuronal activation has similarities to electrical stimulation which has been shown experimentally to transform type II fibres to type I. In the biopsies we found "promiscuous fibres" with various degrees of combined staining for anti-fast and anti- slow MHC. The majority of the fibres were of type I but some were of ATPase IM and some were of type II according to the myofibrillary ATPase stainability. The various degrees of staining for slow and fast MHC in individual fibres support the view that a transformation of fibres has taken place in analogy with the findings in exercised athletes.

Experimental denervation studies have implied that only fast myosin is synthesised in muscle fibres following denervation and studies in rat diaphragms has shown that after eight weeks of denervation, all fibres react strongly with anti-fast myosin and many with anti-slow myosin as well. ${ }^{34}$ Single motor unit experiments have shown that myosin from fast-twitch muscle fibres reinnervated by slow motoneurons convert completely from the fast to their slow isoform..$^{35} \mathrm{It}$ may be argued that the appearance of promiscuous fibres with stainability for both anti-slow MHC and anti-fast MHC may be due to denervation-reinnervation. However, the lack of atrophy within the group of promiscuous fibres indicates that they were innervated.

A further support for a muscle fibre transformation is the finding of highly atrophic fibres which constituted a mixture of type I and type II. In animal experiments it has been shown that after cessation of artificial stimulation, newly created slow-twitch (type I) fibres seemed to revert to their original characteristics. ${ }^{36}$ Furthermore, Larsson and Ansved ${ }^{31}$ showed that during detraining in athletes, the number of type II fibres increased and type I fibres decreased. Since the atrophic fibres should be inactivated due to denervation, type I fibres transformed from type II would revert to their original characteristics. If there was an ongoing selective loss of type II fibres due to denervation one would expect the atrophic fibres to be of type II.

In conclusion, we suggest that motoneurons which originally innervated type II fibres, because of excessive overuse later innervate fibres which have achieved type I fibre characteristics.

This study was supported by grants from the Swedish Medical Research Council (12X-3875, 14X-7479, 12X-3934), the Swedish Association for Traffic and Polio Disabled, the Norrbacka-Eugenia Foundation and the Karolinska Institute.

\section{References}

1 Jakobsson F, Borg K, Edström L, Grimby L. Use of motor units in relation to muscle fiber type and size in man. Muscle Nerve 1988;11:1211-18.

2 Borg K, Borg J, Edström L, Grimby L. Effects of excessive use of remaining muscle in prior polio and LV lesion. Muscle Nerve 1988;11:1219-30.

3 Edström L, Grimby L. Effect of exercise on the motor unit. Muscle Nerve 1986;9:104-26. 
4 Sréter FA, Gergely J, Salmons S, Romanul FCA. Synthesis by fast muscle of myosin characteristic of slow muscle in response to long-term stimulation. Nature (New Biol) 1973;241:17-9.

5 Brown WE, Salmons S, Whalen RG. The sequential replacement of myosin subunit isoforms during muscle type transformation induced by long term electrical stimulation. J Biol Chem 1983 ; 258:686-92.

6 Pette D, Müller W, Leisner E, Vrbová G. Time dependent effects of contractile properties, fibre population, myosin light chains and enzymes of energy metabolism in intermittently and continuously stimulated fast twitch muscle of the rabbit. Pfluegers Arch 1976;364:103-12.

7 Hannerz J. An electrode for recording single motor unit activity during strong muscle contractions. Electroencephalogr Clin Neurophysiol 1974;37:179-81.

8 Grimby L. Firing properties of single human motor units during locomotion. J Physiol (Lond) 1984;346:195-202.

9 Kimura J, Bosch P, Lindsay GM. F-wave conduction velocity in the central segment of the peroneal and tibial nerves. Arch Phys Med Rehabil 1975;56:492-7.

10 Borg J, Grimby L, Hannerz J. Axonal conduction velocity and voluntary discharge properties of individual short toe extensor motor units in man. $J$ Physiol (Lond) 1978;277:143-52.

11 Radner S. Knappnảsteknik för iterativ muskelbiopsi. Transactions of the Swedish Society for Medical Science 1962;XIV:94.

12 Lindholm T. The influence of uraemia and electrolyte disturbances on muscle action potentials and motor nerve conduction in man. Acta Med Scand 1968;Suppl 491.

13 Padykula HA, Herman E. The specificity of the histochemical method of adenosine triphosphatase. J Histochem Cytochem 1955;3:170-83.

14 Brooke MH, Kaiser KK. Muscle fiber types-How many and what kind? Arch Neurol 1970;23:369-79.

15 Schantz PG, Dhoot GK. Co-existence of slow and fast isoforms of contractile and regulatory proteins in human skeletal muscle fibres induced by endurance training. Acta Physiol Scand 1987; 131:147-54.

16 Dhoot GK, Dransfield I, Grand RJA, Perry SV. Distribution of isoforms of the myofibrillar proteins in myoid cells of thymus. $J$ Muscle Res Cell Motil 1986;7:351-60.

17 Sternberger LA. Immunohistochemistry. New York: Wiley Medical, 1979.

18 Grimby $L$. Single motor unit discharge during voluntary contraction and locomotion. In: Jones NL, ed. Human Muscle Power. Chicago: Human Kinetic Publishers Inc, 1986:111-30.

19 Grimby L, Hannerz J, Hedman B. Contraction time and voluntary discharge properties of individual short toe extensor motor units in man. J Physiol (Lond) 1979;289:191-201.

20 Borg K, Borg J. Conduction velocity and refractory period of single motor nerve fibres in antecedent polio-myelitis. J Neurol
Neurosurg Psychiatry 1987;50:443-6.

21 Huizar P, Kuno M, Kudo N, Miyata Y. Reaction of intact spinal motoneurones to partial denervation of the muscle. J Physiol (Lond) 1977;265:175-91.

22 Blintziger K, Kreutzenberg G. Displacement of synaptic terminals from regenerating motoneurones by microglial cells. $Z$ Zellforsch mikrosk Anat 1968;85:145-57.

23 Fujita Y, Harada H, Kitamura T, Minami S, Sato T. Dendritic activities of spinal motoneurones in pigs and rabbits enhanced through chronic stimulation of a dorsal root. J Physiol (Lond) 1987;383:171-90.

24 Bigland-Ritchie B, Woods JJ. Changes in muscle contractile properties and neural control during human muscular fatigue. Muscle Nerve 1984;7:691-99.

25 Edds MV. Hypertrophy of nerve fibers to functionally overloaded muscles. J Comp Neurol 1950;93:259-75.

26 Eisen AA, Carpenter S, Karpati G, Bellavance A. The effect of muscle hyper- and hypoactivity upon fibre diameters of intact and regenerating nerves. $J$ Neurol Sci 1973;20:457-69.

27 Tomanek. RJ, Tipton CM. Influence of exercise and tenectomy on the morphology of a muscle nerve. Anat Rec 1976;159:105-12.

28 Hudlicka O, Tyler KR, Srihari T, Heilig A, Pette D. The effect of different patterns of long-term stimulation on contractile properties and myosin light chains in rabbit fast muscles. Pfluegers Arch 1982;393:164-70.

29 Kernell D, Eerbeek O, Sonselaar Y, Verhey BA. Effects of moderate amounts of fast and slow rates of chronic stimulation on the contractile properties of a fast hindlimb muscle in the cat. Proc Int Union Physiol Sci 1983;15:189.

30 Jean DH, Albers RW, Guth L, Aron HJ. Differences between the heavy chains of fast and slow muscle myosin. Exp Neurol 1975, 49:750-7.

31 Larsson L, Ansved T. Effects of long-term physical training and detraining on enzyme histochemical and functional skeletaf muscle characteristics in man. Muscle Nerve 1985;8:714-22.

32 Schantz PG, Billeter R, Henriksson I, Jansson E. Traininginduced increase in myofibrillar ATPase intermediate fibers in human skeletal muscle. Muscle Nerve 1982;5:628-36.

33 Schantz PG, Henriksson J. Increase in myofibrillar ATPase intermediate muscle fibers in response to endurance training Muscle Nerve 1983;6:553-6.

34 Gauthier GF, Hobbs AW. Effects of denervation on the distribution of myosin isozymes in skeletal muscle fibers. Exp Neurol 1982;76:331-44.

35 Gauthier GF, Burke RE, Lowey S, Hobbs AW. Myosin isozymes in normal cross-reinnervated cat skeletal muscle fibers. $J$ Cell Biol 1983;97:756-64.

36 Salmons S, Sréter FA. Significance of impulse activity in the transformation of skeletal muscle type. Nature 1976;263:30-4. 